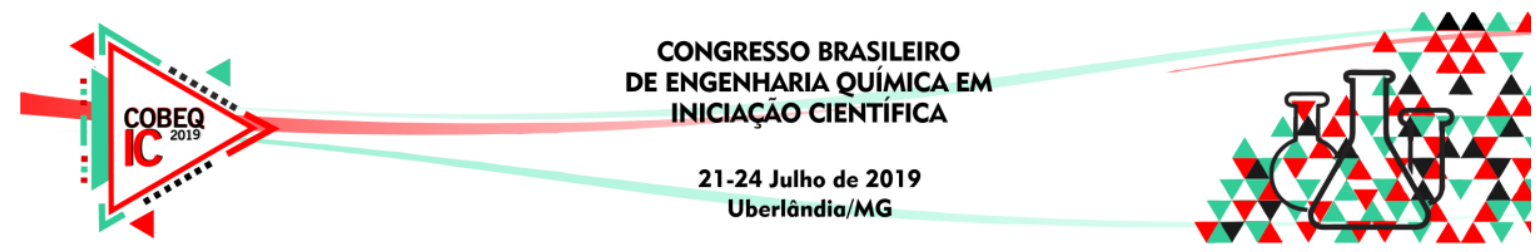

\title{
MODELOS MATEMÁTiCOS AJUSTAdOS A CINÉTICA DE SECAGEM DE MÍNERIOS DE FERRO E CROMO
}

\author{
O. B. Matos $^{1 *}$, A. F. B. Ferreira ${ }^{2}$,M. D. C. Almeida ${ }^{1}$ e P. H. F. Araújo ${ }^{1}$ \\ ${ }^{1}$ Universidade do Estado do Amapá, Colegiado de Engenharia Química, Macapá-AP, Brasil \\ ${ }^{2}$ Universidade Federal do Ceará, Departamento de Engenharia Química, Fortaleza-CE, Brasil \\ *E-mail para contato: tonny-barbosa@hotmail.com
}

\begin{abstract}
RESUMO - O objetivo do presente trabalho é estudar a secagem dos minérios de ferro e cromo - oriundos da Cidade de Santana, Estado do Amapá, região Norte do Brasil - para avaliação da influência da temperatura e de modelos matemáticos de secagem. Os experimentos foram realizados em uma estufa por quase 3 horas e em temperaturas de 100 a $200^{\circ} \mathrm{C}$. Os modelos foram ajustados à secagem experimental com auxílio do software STATISTICA e adotando-se o método de mínimos quadrados para analisar qual modelo melhor se assemelha aos dados experimentais, comparando os valores de coeficiente de determinação $\left(R^{2}\right)$, de erro médio relativo $(E M R)$, de erro médio estimado $(E M E)$ e da tendência de distribuição dos resíduos. A partir dos resultados obtidos, o modelo de aproximação de difusão foi o melhor para o minério de ferro (em todas as temperaturas) e de cromo (em T $=150$ e 200 ${ }^{\circ} \mathrm{C}$ ). Para a secagem do minério de cromo a $100{ }^{\circ} \mathrm{C}$, o melhor modelo foi o de Henderson e Pabis. Ambos os modelos matemáticos obtiveram $R^{2}$ superior a $99,7 \%, E M R$ inferior a $10 \%$ e tendência tipo aleatória.
\end{abstract}

\section{INTRODUÇÃO}

O Brasil é um dos países com maiores reservas de minério do mundo, apresentando rochas contendo hematita $\left(\mathrm{Fe}_{2} \mathrm{O}_{3}\right)$, magnetita $\left(\mathrm{Fe}_{3} \mathrm{O}_{4}\right)$ e cromita $\left(\mathrm{FeO} \cdot \mathrm{Cr}_{2} \mathrm{O}_{3}\right)$. Segundo Carvalho (2014), o minério de ferro é um desses tipos de minério, que vem sendo utilizado na indústria de cimento, em estradas, na produção de veículos e fios elétricos. Mas, sobretudo, em siderurgias (mais de 90\%), pois é a partir dele que se obtém o Ferro metálico para produção do aço. Já a cromita ou minério de cromo é usado para produção de ligas de ferro-cromo, aço inoxidável e ligas especiais por propiciar maior resistência à oxidação, a corrosão e a fadiga (GONÇALVES, 2001). Uma das etapas importantes do beneficiamento de qualquer minério é a secagem, que pode interferir na comercialização e nas suas características devido a granulometria, umidade, teor de ferro e impurezas associadas ao material, uma vez que o minério fica muito tempo exposto às intempéries climáticas. Desta forma, o objetivo deste trabalho é estudar a secagem dos minérios de ferro e de cromo oriundos do Estado do Amapá, para avaliar a secagem sob a faixa de temperatura de 100 a $200{ }^{\circ} \mathrm{C}$ e também para predizer qual modelo matemático melhor se ajusta aos dados experimentais da secagem dos minérios. 


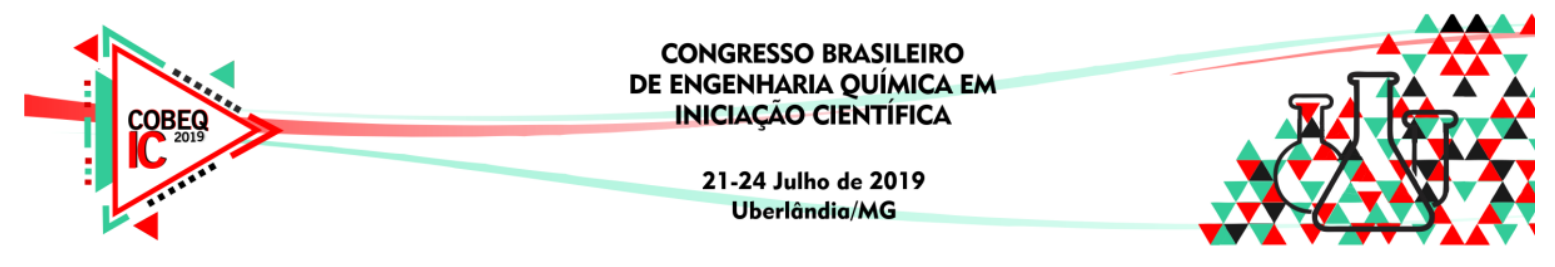

\section{METODOLOGIA}

Os experimentos foram realizados na Universidade do Estado do Amapá-UEAP. As amostras foram obtidas a partir de uma mina presente na cidade de Santana, que está localizada a $20 \mathrm{~km}$ do centro da capital. A metodologia recomendada pela ASABE (2010) foi usada para determinar o teor de água de equilíbrio presente nas amostras. Ambos os minérios de ferro e cromo foram acondicionados em uma estufa e mantidos a $103 \pm 2{ }^{\circ} \mathrm{C}$ por 24 horas. Após a obtenção do teor de água, a secagem foi realizada em uma estufa modelo 403/5 N, marca Nova Ética, nas temperaturas de 100,150 e $200{ }^{\circ} \mathrm{C}$. Para o minério de ferro, a média das massas secadas foram $10,013 \mathrm{~g}\left(100{ }^{\circ} \mathrm{C}\right), 10,002 \mathrm{~g}\left(150{ }^{\circ} \mathrm{C}\right)$ e $10,005 \mathrm{~g}\left(200{ }^{\circ} \mathrm{C}\right)$ e para o minério de cromo as médias das massas foram: $10,28 \mathrm{~g}\left(100{ }^{\circ} \mathrm{C}\right), 10,002 \mathrm{~g}\left(150{ }^{\circ} \mathrm{C}\right)$ e $10,001 \mathrm{~g}\left(200{ }^{\circ} \mathrm{C}\right)$. Ressalta-se que as secagens foram feitas em triplicata.

Durante o processo de secagem é necessário conhecer parâmetros para construir as isotermas de secagem e compreender melhor o processo. Assim, avaliou-se a umidade dos minérios em base seca $\left(X_{b s}\right.$ - Equação 1$)$, em base úmida $\left(X_{b u}\right.$ - Equação 2$)$ e pela razão de umidade ( $R U$ - Equação 3), pois as partículas que os compõem não apresentam formas/tamanhos iguais.

$$
\begin{aligned}
& X_{b s}=\frac{M_{w}}{M_{S C}} \\
& X_{b u}=\frac{M_{w}}{M_{t}} \\
& R U=\frac{X-X_{e}}{X_{o}-X_{e}}
\end{aligned}
$$

onde: $M_{w}$ é a massa de água em um dado tempo de secagem; $M_{s c}$ equivale a massa seca após um período de 24 horas; $M_{w}$ é a diferença entre a massa secada em um dado instante t $\left(M_{t}\right)$ menos a massa da amostra seca final $\left(M_{s c}\right)$ ou massa de equilíbrio; $X$ representa a umidade absoluta; $X_{e}$ é a umidade em equilíbrio e $X_{o}$ é a umidade inicial.

Modelos matemáticos (Equações 4-8) foram ajustados a razão de umidade experimental em função do tempo, utilizando o software STATISTICA ${ }^{\circledR}$ e adotando-se o método de mínimos quadrados para obtenção dos dados de regressão.

$$
\begin{aligned}
& R U=\exp (-k t) \\
& R U=\exp \left(-k t^{n}\right)
\end{aligned}
$$




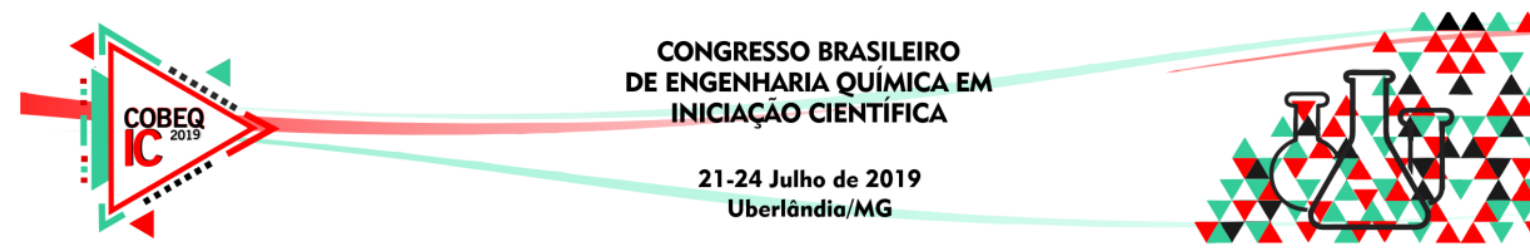

$R U=\operatorname{aexp}(-k t)$

$R U=a \exp (-k t)+(1-a) \exp (-k b t)$

$R U=a \exp (-k t)+(1-a) \exp (-k a t)$

(4) Newton; (5) Page; (6) Henderson e Pabis; (7) Aproximação de difusão; (8) Exponencial de dois termos.

\section{RESULTADOS E DISCUSSÕES}

Os dados obtidos na secagem dos minérios (média da triplicata), em diferentes temperaturas, são apresentados na Figura 1.

Figura 1 - Massas dos minérios de ferro e cromo versus o tempo de secagem.

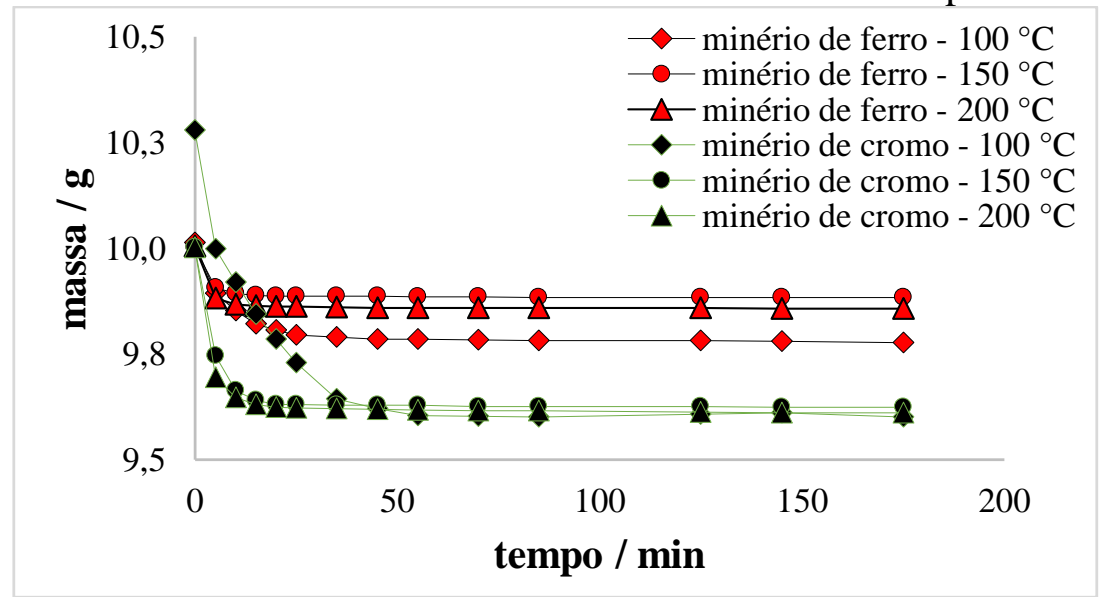

Para todas as temperaturas, os dados experimentais da Figura 1 concordam com o padrão observado por Geankoplis (1998). O incremento da temperatura provoca uma maior retirada de água e os minérios perdem mais umidade no início da secagem, já que a quantidade de água livre é maior na superfície das amostras do que no seio delas. As massas dos minérios precisam de pouco tempo para se estabilizarem (não possuem material orgânico), bem diferente de biomassas e outros alimentos. Nas Tabelas 3-8 são reportados os dados obtidos com os ajustes dos modelos em relação ao coeficiente de determinação $\left(R^{2}\right)$, ao erro médio relativo $(E M R)$, ao erro médio estimado $(E M E)$ e ao tipo de tendência observada das distribuições dos resíduos. 
Tabela 3 - Valores de $R^{2}, E M R, E M E$ e a tendência para o minério de ferro a $\mathrm{T}=100{ }^{\circ} \mathrm{C}$.

\begin{tabular}{cccccc}
\hline Modelo & $\begin{array}{c}\text { Coeficientes e } \\
\text { constantes }\end{array}$ & $\boldsymbol{R}^{\mathbf{2}}(\boldsymbol{\%})$ & $\boldsymbol{E M R}(\boldsymbol{\%})$ & $\boldsymbol{E M E}$ & Tendência \\
\hline Newton & $k=0,9948$ & 99,4793 & 48,5212 & 28,3327 & Tendenciosa \\
\hline Pages & $k=0,2094, n=0,75775$ & 99,8681 & 21,9605 & 4,9976 & Tendenciosa \\
\hline $\begin{array}{c}\text { Henderson e } \\
\text { Pabis }\end{array}$ & $a=0,9761, k=0,1147$ & 99,5101 & 45,5672 & 25,8252 & Tendenciosa \\
\hline $\begin{array}{c}\text { Aproximação } \\
\text { de difusão }\end{array}$ & $\begin{array}{c}\boldsymbol{a}=\mathbf{0 , 8 8 3 1 ,} \boldsymbol{k}=\mathbf{1 4 9 9}, \\
\boldsymbol{b}=\mathbf{0 , 1 5 1 6}\end{array}$ & $\mathbf{9 9 , 8 7 8 5}$ & $\mathbf{7 , 3 7 7 1}$ & $\mathbf{0 , 5 5 9 0}$ & Aleatória \\
\hline $\begin{array}{c}\text { Exponencial } \\
\text { de dois termos }\end{array}$ & $a=0,2540, k=0,3599$ & 99,7980 & 25,0962 & 10,1487 & Tendenciosa \\
\hline
\end{tabular}

Tabela 4 - Valores de $R^{2}, E M R, E M E$ e a tendência para o minério de ferro a $\mathrm{T}=150{ }^{\circ} \mathrm{C}$.

\begin{tabular}{|c|c|c|c|c|c|}
\hline Modelo & $\begin{array}{c}\text { Coeficientes e } \\
\text { constantes }\end{array}$ & $R^{2}(\%)$ & $\operatorname{EMR}(\%)$ & $E M E$ & Tendência \\
\hline Newton & $k=0,3069$ & 99,5740 & 99,2752 & 98,5599 & Tendenciosa \\
\hline Pages & $k=0,8201, n=0,4552$ & 99,9047 & 47,6621 & 22,9497 & Tendenciosa \\
\hline $\begin{array}{l}\text { Henderson e } \\
\text { Pabis }\end{array}$ & $a=0,9973, k=0,3063$ & 99,5744 & 99,2669 & 98,5436 & Tendenciosa \\
\hline $\begin{array}{l}\text { Aproximação } \\
\text { de difusão }\end{array}$ & $\begin{array}{c}a=0,9408, k=0,3763 \\
b=0,0527\end{array}$ & 99,9799 & 12,3596 & 1,5283 & Aleatória \\
\hline $\begin{array}{c}\text { Exponencial de } \\
\text { dois termos }\end{array}$ & $a=0,3681, k=0,6294$ & 99,6806 & 96,8496 & 93,8653 & Tendenciosa \\
\hline
\end{tabular}

Tabela 5 - Valores de $R^{2}, E M R, E M E$ e a tendência para o minério de ferro a $\mathrm{T}=200{ }^{\circ} \mathrm{C}$.

\begin{tabular}{cccccc}
\hline Modelo & $\begin{array}{c}\text { Coeficientes e } \\
\text { constantes }\end{array}$ & $\boldsymbol{R}^{\mathbf{2}(\%)}$ & $\boldsymbol{E M R}(\boldsymbol{\%})$ & $\boldsymbol{E M E}$ & Tendência \\
\hline Newton & $k=0,3439$ & 99,6530 & 98,2019 & 96,4518 & Tendenciosa \\
\hline Pages & $k=0,9623, n=0,4155$ & 99,9572 & 25,7458 & 6,6506 & Tendenciosa \\
\hline $\begin{array}{c}\text { Henderson e } \\
\text { Pabis }\end{array}$ & $a=0,9983, k=0,3435$ & 99,6531 & 98,1919 & 96,4324 & Tendenciosa \\
\hline $\begin{array}{c}\text { Aproximação } \\
\text { de difusão }\end{array}$ & $\boldsymbol{a}=\mathbf{0 , 9 3 1 1 5 ,} \boldsymbol{k}=\mathbf{0 , 4 3 7 7 ,}$ & $\mathbf{9 9 , 9 9 3 1}$ & $\mathbf{9 , 0 2 6 9}$ & $\mathbf{0 , 8 2 5 3}$ & Aleatória \\
\hline $\begin{array}{c}\text { Exponencial } \\
\text { de dois termos }\end{array}$ & $a=0,3875, k=0,6716$ & 99,7296 & 93,6637 & 87,8603 & Tendenciosa \\
\hline
\end{tabular}




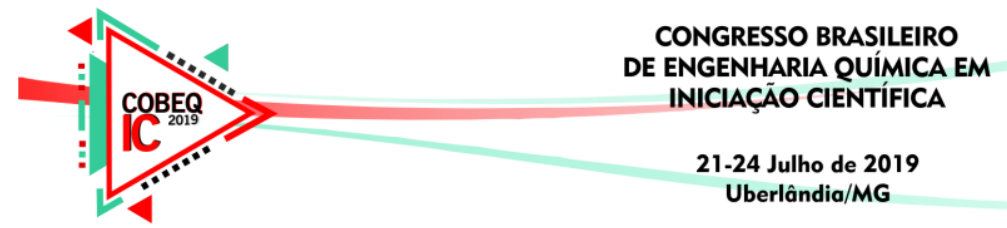

Tabela 6 - Valores de $R^{2}, E M R, E M E$ e a tendência para o minério de cromo a T $=100{ }^{\circ} \mathrm{C}$.

\begin{tabular}{cccccc}
\hline Modelo & $\begin{array}{c}\text { Coeficientes e } \\
\text { constantes }\end{array}$ & $\boldsymbol{R}^{\mathbf{2}(\boldsymbol{\%})}$ & $\boldsymbol{E M R}(\boldsymbol{\%})$ & $\boldsymbol{E M E}$ & Tendência \\
\hline Newton & $k=0,0738$ & 99,3238 & 17,5309 & 3,0772 & Aleatória \\
\hline Pages & $k=0,8376, n=0,1162$ & 99,6001 & 34,7627 & 17,3002 & Tendenciosa \\
\hline $\begin{array}{c}\text { Henderson e } \\
\text { Pabis }\end{array}$ & $\boldsymbol{a}=\mathbf{0 , 9 5 6 0 ,} \boldsymbol{k}=\mathbf{0 , 0 7 0 2}$ & $\mathbf{9 9 , 4 2 9 1}$ & $\mathbf{2 0 , 7 5 5 5}$ & $\mathbf{4 , 6 4 1 8}$ & Aleatória \\
\hline $\begin{array}{c}\text { Aproximação } \\
\text { de difusão }\end{array}$ & $\begin{array}{c}a=0,1766, k=2,1641, \\
b=0,0276\end{array}$ & 99,7822 & 32,7542 & 16,6501 & Tendenciosa \\
\hline $\begin{array}{c}\text { Exponencial } \\
\text { de dois termos }\end{array}$ & $a=0,1432, k=0,4407$ & 99,6980 & 28,1785 & 10,9554 & Tendenciosa \\
\hline
\end{tabular}

Tabela 7 - Valores de $R^{2}, E M R, E M E$ e a tendência para o minério de cromo a T $=150{ }^{\circ} \mathrm{C}$.

\begin{tabular}{cccccc}
\hline Modelo & $\begin{array}{c}\text { Coeficientes e } \\
\text { constantes }\end{array}$ & $\boldsymbol{R}^{\mathbf{2}}(\boldsymbol{\%})$ & $\boldsymbol{E M R}(\boldsymbol{\%})$ & $\boldsymbol{E M E}$ & Tendência \\
\hline Newton & $k=0,2223$ & 99,9347 & 89,5380 & 80,7824 & Tendenciosa \\
\hline Pages & $k=0,2598, n=0,9176$ & 99,9461 & 80,1641 & 65,8713 & Tendenciosa \\
\hline $\begin{array}{c}\text { Henderson e } \\
\text { Pabis }\end{array}$ & $a=0,9988, k=0,2221$ & 99,9348 & 89,4965 & 80,7123 & Tendenciosa \\
\hline $\begin{array}{c}\text { Aproximação } \\
\text { de difusão }\end{array}$ & $\begin{array}{c}\boldsymbol{a}=\mathbf{0 , 9 7 8 8 ,} \boldsymbol{k}=\mathbf{0 , 2 3 5 7}, \\
\boldsymbol{b}=\mathbf{0 , 0 5 6 4}\end{array}$ & $\mathbf{9 9 , 9 9 4 3}$ & $\mathbf{1 4 , 5 4 9 0}$ & $\mathbf{2 , 1 1 8 8}$ & Aleatória \\
\hline $\begin{array}{c}\text { Exponencial } \\
\text { de dois termos }\end{array}$ & $a=0,5442, k=0,3013$ & 99,9507 & 76,7280 & 60,8089 & Tendenciosa \\
\hline
\end{tabular}

Tabela 8 - Valores de $R^{2}, E M R, E M E$ e a tendência para o minério de cromo a $\mathrm{T}=200{ }^{\circ} \mathrm{C}$.

\begin{tabular}{|c|c|c|c|c|c|}
\hline Modelo & $\begin{array}{c}\text { Coeficientes e } \\
\text { constantes }\end{array}$ & $R^{2}(\%)$ & $\operatorname{EMR}(\%)$ & $E M E$ & Tendência \\
\hline Newton & $k=0,2841$ & 99,5847 & 98,5755 & 97,1869 & Tendenciosa \\
\hline Pages & $k=0,6957, n=0,5095$ & 99,9302 & 36,0602 & 14,8582 & Tendenciosa \\
\hline $\begin{array}{l}\text { Henderson e } \\
\text { Pabis }\end{array}$ & $a=0,9963, k=0,2834$ & 99,5855 & 98,5532 & 97,1436 & Tendenciosa \\
\hline $\begin{array}{c}\text { Aproximação } \\
\text { de difusão }\end{array}$ & $\begin{array}{c}a=0,9301, k=0,3547 \\
b=0,0718\end{array}$ & $\mathbf{9 9 , 9 7 8 2}$ & 5,9973 & 0,4058 & Aleatória \\
\hline $\begin{array}{l}\text { Exponencial de } \\
\text { dois termos }\end{array}$ & $a=0,3567, k=0,6000$ & 99,7191 & 94,3902 & 89,2829 & Tendenciosa \\
\hline
\end{tabular}

Os resultados das Tabelas 3-8 mostram que todos os modelos matemáticos apresentam $R^{2}$ superior a 99\%. De acordo com Madamba et al. (1996), $R^{2}$ acima de $99,9 \%$ indica os melhores modelos de secagem. Para as amostras de ferro, verifica-se que o modelo de Aproximação de difusão se ajustou muito bem aos dados experimentais da secagem do minério de ferro, em todas as temperaturas, pois apresentou $R^{2}$ superior a $99,9 \%, E M R$ menor do que $10 \%$ e baixo $E M E$. O mesmo modelo também foi o melhor para ajustar praticamente quase todos os dados do minério de cromo, com exceção dos dados a $\mathrm{T}=100{ }^{\circ} \mathrm{C}$, na qual o melhor modelo foi o Henderson e Pabis. A Figura 2 ilustra os dados experimentais e preditos para $R U$, através dos melhores modelos expostos nas Tabelas 3-8 em cada temperatura estudada. 


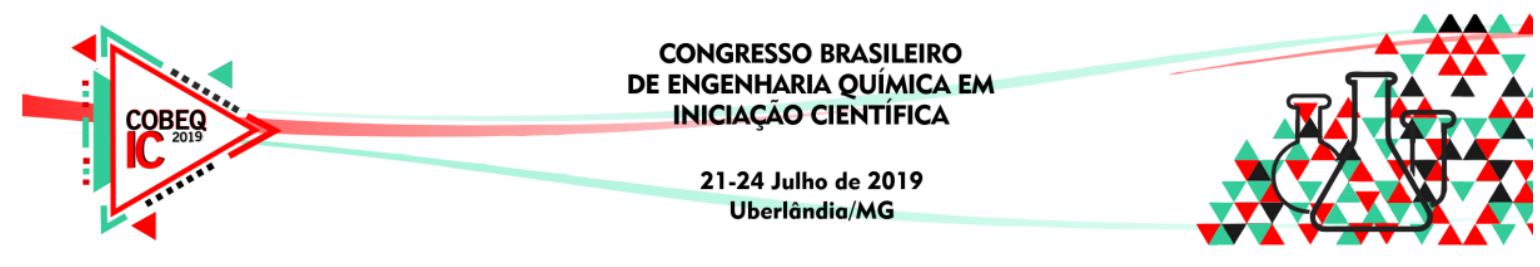

Figura 2 - RU dos valores experimentais e preditos para os minérios de ferro e cromo.

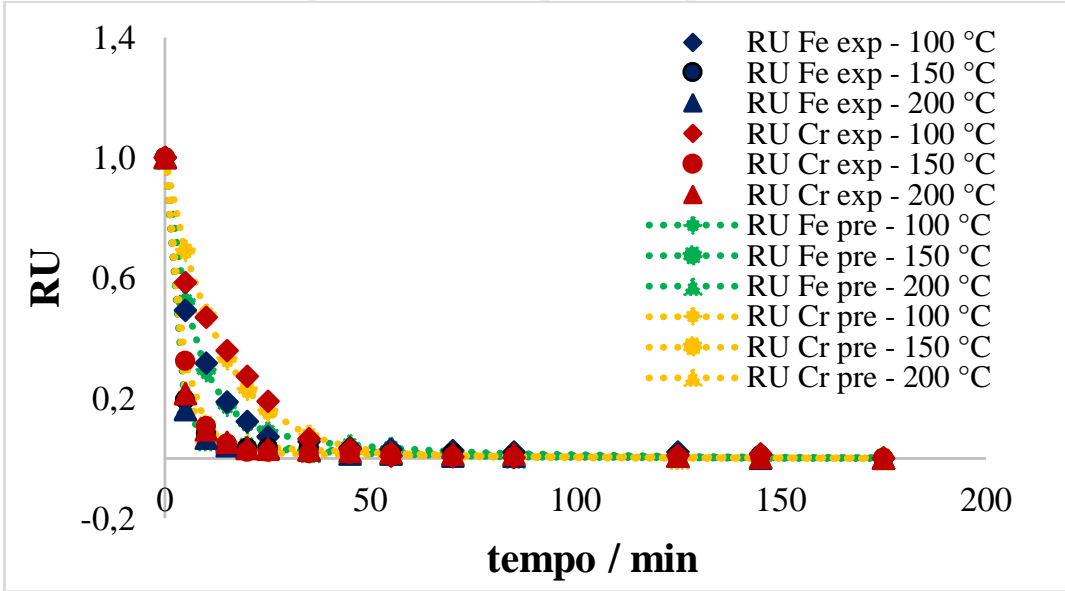

Pela Figura 2, nota-se que o modelo de aproximação de difusão apresenta comportamento similar aos dados experimentais da secagem do minério de ferro (em todas as temperaturas avaliadas) e de cromo ( $\mathrm{T}=150$ e $\left.200^{\circ} \mathrm{C}\right)$. Já o modelo de Henderson e Pabis também concorda satisfatoriamente com os valores experimentais do minério de cromo a $\mathrm{T}=100{ }^{\circ} \mathrm{C}$.

\section{CONCLUSÃO}

Percebeu-se que todos os modelos usados para ajustar os dados experimentais apresentaram $R^{2}>99 \%$. Mas, o modelo de Aproximação de difusão foi o mais eficiente para os minérios estudados. Ainda assim, o modelo de Henderson e Pabis se assemelhou aos dados experimentais do minério de cromo a $\mathrm{T}=100^{\circ} \mathrm{C}$. Por fim, faz-se necessário a continuidade do trabalho com outros estudos futuros, a fim de caracterizar de forma físico-química as amostras utilizadas e confrontar com os padrões industriais validados.

\section{REFERÊNCIAS}

ASABE - American Society of Agricultural and Biological Engineers. Moisture Measurement - Forages: Standard S358.2 DEC1988, R2008. American Society of Agricultural and Biological Engineers (ed.). Standards, Engineering Practices, and Data. St. Joseph: ASABE, p.684-685, 2010.

CARVALHO, P. S. L. Minério de ferro. BNDES: biblioteca digital, p. 197-234, 2014.

GEANKOPLIS, C. J. Procesos de transporte y operaciones unitarias. 3.ed. Cidade do México: Compãnía Editorial Continental, 1998.

GONÇALVES, M. M. Balanço Mineral Brasileiro. 2001. Disponível em: $<$ http://www.dnpm.gov.br/dnpm/paginas/balanco-mineral/arquivos/balanco-mineralbrasileiro-2001-cromo>. Acesso em: 13 de abril de 2019.

MADAMBA, P. S.; BUCKLE, K. A.; DRISCOLL, R. H. The thin-layer drying characteristics of garlic slices. Journal of Food Engineering, v.29, p. 75-97, 1996. 